\title{
A SIMPLE ANALYTICAL METHOD FOR DETERMINING BASIC HYDRODYNAMIC CHARACTERISTICS OF HYBRID FLUIDIZED-BED AIR-LIFT APPARATAE
}

\author{
Bolesław Tabiś*, Dominika Boroń \\ Cracow University of Technology, Department of Chemical and Process Engineering, \\ ul. Warszawska 24, 30-155 Kraków, Poland
}

\begin{abstract}
A simple analytical method for determination of basic hydrodynamic characteristics of hybrid fluidized-bed air-lift devices was presented. These devices consist of two parts: a two-phase air-lift part and a two-phase liquid-solid fluidized-bed part. Forced circulation of fluid in the air-lift part is used for fluidization of solid particles in the fluidized-bed part. According to the opinion given in the literature, if such apparatus is used for aerobic microbiological processes, its advantage is lower shear forces acting on the biofilm immobilized on fine-grained material compared with shear forces in three-phase fluidized-bed bioreactors. Another advantage is higher biomass concentration due to its immobilization on fine particles, compared with two-phase airlift bioreactors. A method of calculating gas hold-up in the air-lift part, and gas and liquid velocities in all zones of the analyzed apparatus is presented.
\end{abstract}

Keywords: fluidized-bed, hydrodynamics, air-lift, hybrid apparatus, bioreactor

\section{INTRODUCTION}

In aerobic microbiological processes, widespread in biotechnology, several types of bioreactors are used. Tank and column bubble bioreactors are the simplest types of reactor construction. Air-lift devices, both with an external and internal circulating tube, are also classified as bubble bioreactors.

Fluidized-bed two- and three-phase bioreactors have been proposed in the last decades to increase mean biomass concentration inside the apparatus. Utilization of fine particles in fluidized-bed bioreactors facilitates a greater mass transfer surface between the liquid phase and the biofilm. In addition, fluidization prevents bed clogging, which can occur in fixed-bed bioreactors, for example in biofilters.

Immobilization of microorganisms in the fluidized bed has other advantages as well (Mowla and Ahmadi, 2007; Tang and Fan, 1987), such as: i) an increase in the overall microbiological process rate because of high total biomass concentration, ii) a possibility of continuous exchange of bioparticles and supervision of the thickness and age of biofilm, iii) a smaller device, compared with other reactors of the same efficiency.

The advantages of fluidized-bed bioreactors have caused significant spread of this type of devices (Tabiś and Stryjewski, 2013). Fluidized-bed bioreactors usually are three-phase vessels. They can be divided into bioreactors without liquid recirculation (Fig. 1a) and bioreactors with internal circulation used as fluidized-bed air-lift reactors (Fig. 1b-d). Aeration intensity has an essential influence on the 
hydrodynamic characteristics of such bioreactors. A sufficiently high gas flow rate may cause internal circulation of all three phases, i.e. liquid, gas and solid phase (Fig. 1d).
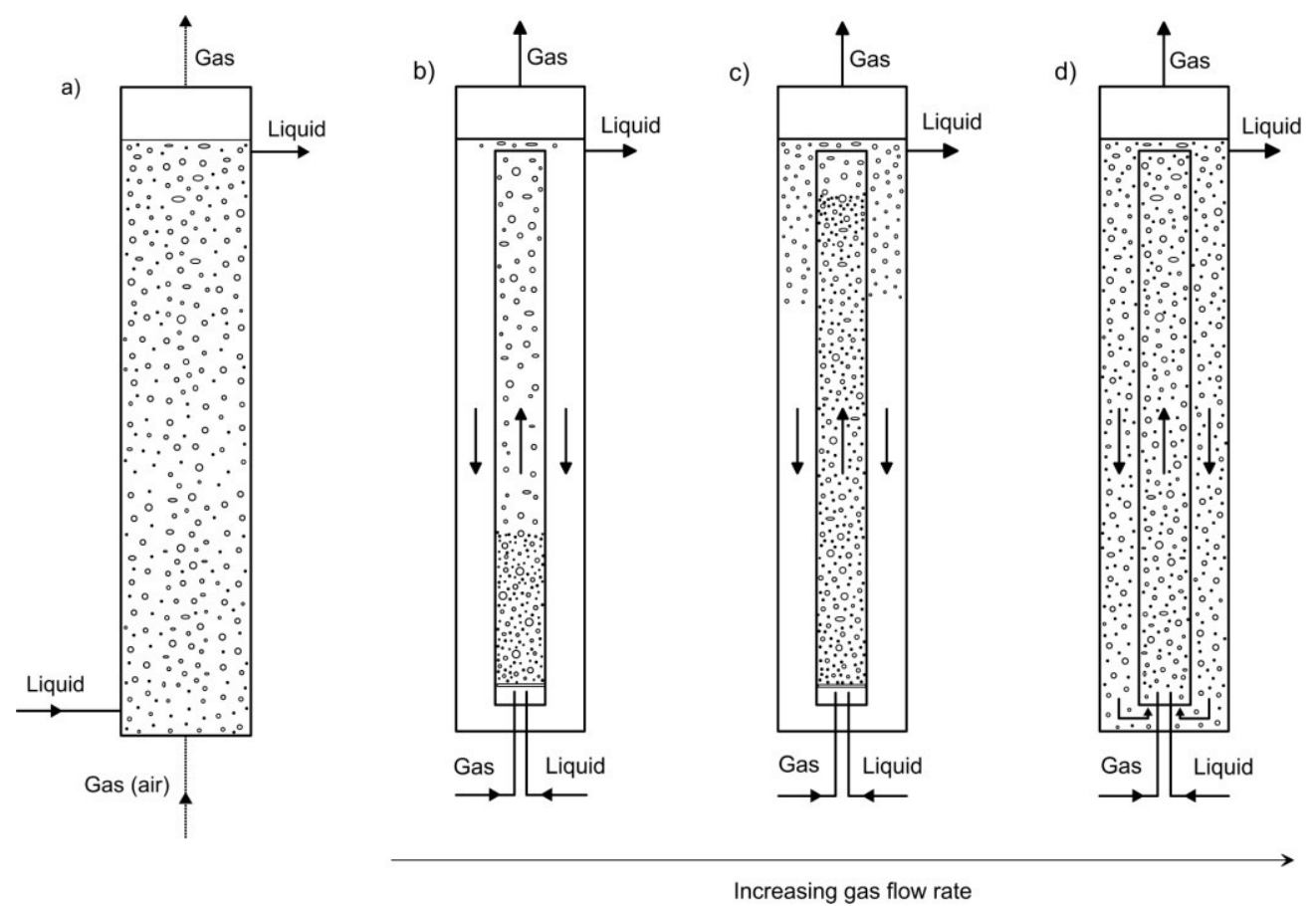

Fig. 1. Two configurations of three-phase bioreactors a) bubble column three-phase bioreactor; b) - d) possible hydrodynamic conditions in a three-phase air-lift bioreactor

Three-phase bioreactors have been the subject of numerous studies in various scientific centers throughout the world. These investigations concerned hydrodynamics (Heijnen et al., 1997; KawalecPietrenko, 2004; Lu, Hwang and Chang, 1995; Merchuk and Siegel, 1988; Merchuk, 2003; Miyahara and Kawate, 1993; Nore et al., 1992; Vuniak-Novakovic et al., 1992), as well as microbiological processes carried out in such devices (Dunn et al, 2003; Huang et al., 1997; Livingstone, 1991; Onysko et al., 2002; Sarra et al., 1997; Tabiś and Georgiou, 2003; Wisecarver and Fan, 1989).

In spite of many advantages mentioned above, three-phase bioreactors have an essential disadvantage. It consists in the existence of shear forces appearing near the gas sparger and in the surrounding of gas bubbles. These forces destroy the biofilm formed on fine particles of inert support. This phenomenon results in a detachment of the biofilm and its drifting with the liquid phase. Guo et al. (1997) and Olivieri et al. (2010) consider this phenomenon as the main disadvantage of three-phase fluidized-bed bioreactors. Therefore a number of scientists analyzed two-phase devices (Dziubiński and Sowiński, 1999; Dziubiński and Sowiński, 2002; Grzywacz, 2013; Kawalec-Pietrenko and Holowacz, 1998). Another question is to separate bioparticles from aeration zone (Dunn et al., 1983; Kawalec-Pietrenko, 2004). There is also a number of publications concerning inverse fluidized-bed airlift reactors. They deal with such issues as hydrodynamics, mass transfer conditions and process parameters (Garnier et al., 1990; Kawalec-Pietrenko and Łazarczyk, 2004; Kawalec-Pietrenko and Matczak, 2006; KawalecPietrenko, 2000). These devices use low density particles. However, particle heavier than liquid phase are also frequently used in microbiological processes.

\section{HYBRID FLUIDIZED-BED AIR-LIFT APPARATUS}

A concept of hybrid fluidized-bed bioreactors with a two phase fluidized bed was proposed and published in the late 1990s (Guo et al., 1997) and then at the beginning of the present century (Olivieri 
et al., 2010). The two fundamental ideas of these hybrid bioreactors are the increase of biomass concentration in comparison with air-lift bioreactors and the separation of the gas phase from the fluidized bed. There are two characteristic components which determine the work mode of the hybrid devices. One part of the apparatus works as a two-phase gas-liquid air-lift device. The other part is a two-phase liquid-solid fluidized-bed vessel. The forced circulation of liquid in the air-lift part maintains fluidization of solid particles in the fluidized-bed part. The hybrid apparatus can operate with external (Fig. 2a), as well as with internal circulation of liquid (Fig. 2b). Figure 2b shows characteristic dimensions used for a quantitative description of the hydrodynamics of the device considered bellow.

The authors have analyzed the hydrodynamic characteristics of the apparatus presented in Fig. $2 \mathrm{~b}$. The gas phase, i.e. air, is supplied at a certain height of the internal circulation tube. The movement of the liquid is caused by density difference between the riser ,„2" and the downcomer „3”. The liquid flows through an annular cross section to the lower part of the apparatus (zone number „1") beneath the perforated bottom. If the velocity of liquid circulation is high enough, solid particles start to fluidize. An additional net beneath the gas sparger retains solid particles in the upper section „2”.

Although hybrid fluidized-bed devices are rather novel and not well-known yet such constructions of the reactors have been used in biotechnology. Up to date, both steady-state and dynamic properties of such bioreactors are unknown. During numerical simulation of such devices working as bioreactors, one has to solve model equations many times, which is a time consuming operation. Thus it will be useful for engineers to have a rather simple analytical model describing the hydrodynamics of such devices. Wherefore, the aim of the work was to propose a simple method for fast estimation of basic hydrodynamic parameters needed for the simulation of hybrid fluidized-bed microbiological reactors. On the basis of the proposed model some hydrodynamic limitations imposed on the operation of such devices may also be determined. The presented results of numerical experiments make the choice of the operating parameters easier. It is important not only for numerical simulations, but also for the engineers who will deal with hybrid fluidized-bed bioreactors in the future.
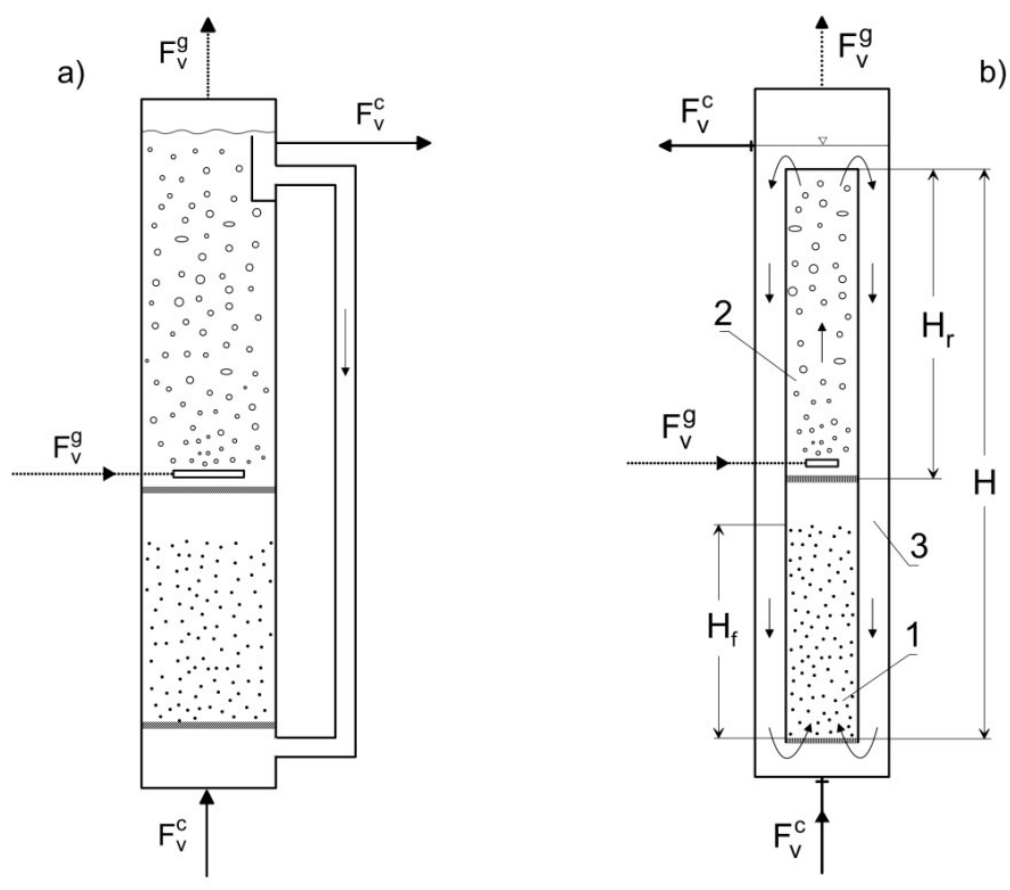

Fig. 2. Two types of hybrid fluidized-bed devices; a) apparatus with external recirculation of the liquid phase; b) apparatus with an internal draft tube 


\section{HYDRODYNAMIC MODEL OF A CONTINUOUS HYBRID FLUIDIZED-BED AIR-LIFT APPARATUS}

In order to model and design a hybrid apparatus (shown in Fig. 2b), the following hydrodynamic quantities are needed: i) superficial liquid velocity in the zone „1", $u_{0 c 1}$, ii) gas hold-up, $\varepsilon_{2}$, and velocity of gas, $u_{g 2}$, in the riser ,2", iii) superficial velocity of liquid in the riser zone, $u_{0 c 2}$, iv) superficial velocity of liquid in the downcomer zone, $u_{0 c 3}$, v) dynamic height of the fluidized bed, $H_{f}$.

The superficial velocity of liquid in the fluidized-bed zone should satisfy the conditions:

$$
u_{m f}<u_{0 c 1}<u_{t}
$$

It is well known that fluidized-bed expansion depends on the velocity of a fluidizing medium. Therefore, we should impose another condition on the superficial liquid velocity in zone „1”, that is:

$$
u_{0 c 1}: H_{f}<H-H_{r}
$$

The superficial liquid velocity in zone „3" should be lower than slip velocity, that was defined by Heijnen et al. (1997). This velocity may be calculated as follows

$$
v=1.53 \cdot\left(\frac{g \sigma\left(\rho_{c}-\rho_{g}\right)}{\rho_{c}^{2}}\right)^{0.25}
$$

The liquid velocity in the downcomer ,3" should satisfy the following condition:

$$
u_{c 3}=u_{0 c 3}<v
$$

The last inequality indicates that gas bubbles do not enter the downcomer. This, in turn, prevents the gas phase from penetrating the fluidized-bed zone.

The density difference between the two-phase medium in the zone „2" and the degassed liquid in the zone ,3" is a driving force needed for liquid circulation between the air-lift zone and the fluidized-bed zone. The pressure difference between both zones can be written as

$$
\Delta p=H_{r} \varepsilon_{2} \rho_{c} g
$$

The driving force of the liquid phase movement at steady state is balanced by the following pressure drops:

i) pressure drops in the internal tube (zone „1" and „2") and the downcomer zone ,3”

$$
\begin{gathered}
\Delta p_{1}=0.5 \lambda_{1} \frac{H-H_{r}}{d_{1}} u_{c 1}^{2} \rho_{c} \\
\Delta p_{2}=0.5 \lambda_{2} \frac{H_{r}}{d_{2}} u_{c 2}^{2} \rho_{c} \\
\Delta p_{3}=0.5 \lambda_{3} \frac{H}{d_{h}} u_{0 c 3}^{2} \rho_{c}, \quad d_{h}=d_{3}-d_{2}
\end{gathered}
$$

ii) pressure drop in the fluidized bed

$$
\Delta p_{f}=H_{m f}\left(\rho_{s}-\rho_{c}\right)\left(1-\varepsilon_{m f}\right) g
$$

iii) pressure drop in the surroundings of the lower edge of the draft tube 


$$
\Delta p_{b}=0.5 \zeta_{b} u_{0 c 3}^{2} \rho_{c}
$$

iv) pressure drop at both nets

$$
\Delta p_{s}=\zeta_{s} u_{c 1}^{2} \rho_{c}
$$

The following formula results from the comparison of the impellent and total pressure drop

$$
\Delta p=\Delta p_{1}+\Delta p_{2}+\Delta p_{3}+\Delta p_{f}+\Delta p_{b}+\Delta p_{s}
$$

A slightly different approach to calculating the pressure balance was proposed by Olivieri et al. (2010). However, it is possible to show that their method gives the same outcomes like the one presented in this work.

In order to calculate the above-mentioned hydrodynamic parameters, i.e. superficial liquid velocity and gas velocity and gas hold-up in zone number „2", one has to complete Eq. (12) with the gas and liquid mass balances:

$$
\begin{gathered}
S_{2} \varepsilon_{2}\left(u_{c 2}+v\right)=S_{2} u_{0 g} \\
S_{2}\left(1-\varepsilon_{2}\right) \cdot u_{c 2}=S_{3} u_{0 c 3}+F_{V}^{c}=S_{3} u_{0 c 3}+S_{2} u_{0 c}
\end{gathered}
$$

At steady-state conditions, liquid velocities in zones „1" and „2" are tied up by the following continuity equation:

$$
S_{1} u_{0 c 1}=S_{2} u_{c 2}\left(1-\varepsilon_{2}\right)
$$

Based on Eqs. (14) and (15), it is possible to calculate the superficial liquid velocities in zones „1” and ,3”, which occur in Eqs. (6), (8), (10) and (11):

$$
\begin{gathered}
u_{0 c 1}=\frac{S_{2}}{S_{1}} u_{c 2}\left(1-\varepsilon_{2}\right) \\
u_{0 c 3}=\frac{S_{2}}{S_{3}}\left[\left(1-\varepsilon_{2}\right) \cdot u_{c 2}-u_{0 c}\right]
\end{gathered}
$$

In this way, the gas hold-up in zone „2", $\varepsilon_{2}$, and the liquid velocity in this zone, $u_{c 2}$, are the only unknown variables. To calculate these quantities, we can use two equations:

$$
\begin{gathered}
\varepsilon_{2} u_{g 2}-u_{0 g}=f_{1}\left(\varepsilon_{2}, u_{c 2}\right)=0 \\
\Delta p-\Delta p_{1}-\Delta p_{2}-\Delta p_{3}-\Delta p_{f}-\Delta p_{b}-\Delta p_{s}=f_{2}\left(\varepsilon_{2}, u_{c 2}\right)=0
\end{gathered}
$$

These are two nonlinear algebraic equations with respect to $\varepsilon_{2}$ and $u_{c 2}$. They can be solved by Newton method, for instance. In this paper, hydrodynamic properties of a hybrid fluidized-bed bioreactor are described by a set of six functions: $u_{0 c 1}\left(u_{0 g}\right), \varepsilon_{2}\left(u_{0 g}\right), u_{g 2}\left(u_{0 g}\right), u_{0 c 2}\left(u_{0 g}\right), u_{0 c 3}\left(u_{0 g}\right), H_{f}\left(u_{0 g}\right)$. These functions were evaluated in a certain range of values $u_{0 g} \geq u_{0 g \text {,min }}$. This range satisfies the conditions (1), (2) and (4), where:

$$
u_{0 g, \min }: u_{0 c 1}=u_{m f}
$$

Condition (2) was verified by calculating the dynamic bed height, $H_{f}$, for each value of $u_{0 g}$, according to empirical relationships

$$
H_{f}=H_{m f} \frac{\left(1-\varepsilon_{m f}\right)}{\left(1-\varepsilon_{f}\right)}
$$


or

$$
\frac{H_{f}}{H_{m f}}=f\left(\frac{u_{0 c 1}}{u_{m f}}\right)
$$

When modeling the hydrodynamics of the hybrid fluidized-bed apparatus, it is important to choose an accurate formula, that allows to calculate the dynamic height of the fluidized bed in the entire range of fluidization ratio, especially for $l_{f}$ close to $l_{f t}$. It is so because inequality (2) has to be fulfilled. In order to verify the existing correlations, experimental measurements of expansion of two-phase liquid-solid beds were performed. The results of these measurements are presented in Fig. 3. Various densities of solid particles were considered. The properties of solids are presented in Table 1. The Morphologi G3, a high-accuracy analyzer of the size and shape of particles was used to obtain geometrical characteristics of solid particles. This microscope with dedicated software determines the diameter and shape factor for every single particle. The result is the distribution of geometrical parameters of a test sample. Furthermore, it is possible to calculate the mean parameters of fine-grained materials. The fluidization agent was tap water. Homogeneous fluidization was observed over the whole range of the fluidization ratio.

Table 1. Properties of analyzed fine-grained materials

\begin{tabular}{|c|c|c|c|}
\hline & Density, $\mathrm{kg} / \mathrm{m}^{3}$ & Range of diameters, $\mathrm{m}$ & $\begin{array}{c}\text { Height of the fluidized bed at minimum } \\
\text { fluidization conditions, } \mathrm{m}\end{array}$ \\
\hline coal & 1412 & $4.25 \cdot 10^{-4}-8.0 \cdot 10^{-4}$ & $0.02 ; 0.04 ; 0.06$ \\
\hline brick & 2384 & $4.25 \cdot 10^{-4}-10^{-3}$ & $0.02 ; 0.04 ; 0.06$ \\
\hline sand & 2620 & $4.25 \cdot 10^{-4}-10^{-3}$ & $0.02 ; 0.04 ; 0.06$ \\
\hline
\end{tabular}

The results of the experiments presented in Fig. 3 show that association of the dynamic bed height solely with the fluidization ratio is rather scanty. For the high fluidization ratio, the experimental data are scattered. This indicates the existence of an additional dependence of the bed height on other parameters.

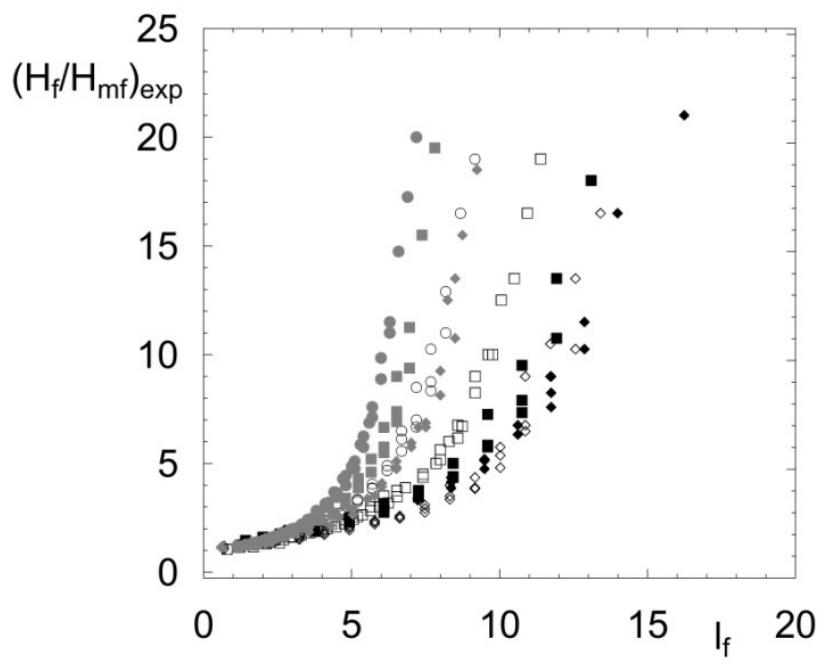

Fig. 3. Experimental data of liquid-solid fluidized-bed expansion $\left(\diamond-\right.$ sand $d_{s}=0.53 \mathrm{~mm}, \diamond-\operatorname{coal} d_{s}=0.53 \mathrm{~mm}$; $\checkmark-$ brick $d_{s}=0.53 \mathrm{~mm} ; \square-$ sand $d_{s}=0.72 \mathrm{~mm}, \boldsymbol{\square}-\operatorname{coal} d_{s}=0.72 \mathrm{~mm}$; - brick $d_{s}=0.72 \mathrm{~mm}$; $\circ-$ sand $d_{s}=0.90 \mathrm{~mm}, \bullet-$ brick $d_{s}=0.90 \mathrm{~mm}$ )

In the literature there exist a lot of empirical correlations that tie porosity or height of a fluidized bed with fluidizing medium velocity and with physicochemical properties of both phases. They are quoted 
in many papers concerning fluidized-bed hydrodynamics, among others, the works of Di Felice (1995), Kmieć (1980), Razumov (1975) and Tripathy et al., (2013).

To calculate a dynamic bed height, one can use the empirical Eq. (21) or more complicated equations, which account for physiochemical properties of the heterogeneous environment

$$
f\left(\frac{H_{f}}{H_{m f}}\right)=\varphi\left(\operatorname{Re}, \operatorname{Ar}, l_{f}, \Phi\right)
$$

Reynolds number Re and Archimedes number Ar in Equation (22a) can be calculated according to formulas (22b) and (22c)

$$
\begin{gathered}
\operatorname{Re}=\frac{u_{0 c} d_{1} \rho_{c}}{\eta_{c}} \\
\operatorname{Ar}=\frac{g d_{s}^{3}\left(\rho_{s}-\rho_{c}\right) \rho_{c}}{\eta_{c}^{2}}
\end{gathered}
$$

A simple, two-parameter correlation to calculate $\varepsilon_{f}$ was proposed by Razumow (1975)

$$
\varepsilon_{f}=1-\frac{1-\varepsilon_{m f}}{a+b l_{f}}
$$

In this formula parameters $a$ and $b$ are experimental values.

Another correlation, published recently by Tripathy (2013), is

$$
\frac{H_{f}}{H_{m f}}=1+6 \cdot 10^{-7} \cdot l_{f}^{1.4046} \cdot\left(\frac{\rho_{c}}{\rho_{s}}\right)^{1.4843}\left(\frac{d_{r}}{d_{s}}\right)^{1.962}
$$

whereas Kmieć (1980) proposed the following empirical formula to calculate $\varepsilon_{f}$

$$
\varepsilon_{f}=1.733 \cdot \operatorname{Re}_{s}^{0.2926} \cdot \mathrm{Ar}^{-0.209}
$$

Reynolds number $\operatorname{Re}_{s}$ in Equation (25a) is defined as

$$
\operatorname{Re}_{s}=\frac{u_{0 c} d_{s} \rho_{c}}{\eta_{c}}
$$

The expansion of the fluidized bed may also be calculated from semi-empirical equations. For example, in order to apply Leva's equation (1959), one has to assume that a fluidized bed is considered to be a fixed bed with changing porosity. Furthermore, while using this equation, one has to assume a constant pressure drop during fluidization with changing velocity of liquid, which is a property of a fluidized bed. Thus, Leva`s equation takes the following from

$$
\lambda \cdot \frac{u_{f}^{2}}{2 d_{s}} \cdot \rho_{c}\left(\frac{\left(1-\varepsilon_{f}\right)^{2-n}}{\varepsilon_{f}{ }^{3}} \cdot \Phi^{3-n}\right)-\left(\rho_{s}-\rho_{c}\right) \cdot g=0
$$

In Eq. (26), empirical dependency $\lambda\left(\operatorname{Re}_{s}, \varepsilon_{f}\right)$ proposed by Ergun (1952) may be applied. In this way, we obtain Eq. (27)

$$
\frac{150 \cdot\left(1-\varepsilon_{f}\right)}{\varepsilon_{f}{ }^{3} \cdot \Phi_{s}{ }^{2}} \cdot \operatorname{Re}_{s}{ }^{2}+\frac{1.75}{\varepsilon_{f}{ }^{3} \cdot \Phi_{s}} \cdot \operatorname{Re}_{s}{ }^{2}-\mathrm{Ar}=0
$$

On the other hand, the streamlined model, presented by Dziubiński and Prywer (2009), results from the balance of forces for a single solid particle. The force of gravity is compared with the sum of buoyancy 
and viscous drag forces. On this basis the following dependency (Dziubiński and Prywer, 2009) was proposed

$$
\varepsilon_{f}^{4.7}=\frac{\left(C_{x}\right)_{u} \cdot A_{s} \cdot \rho_{c} \cdot u_{f}^{2}}{2 \cdot V_{s} \cdot g \cdot\left(\rho_{s}-\rho_{c}\right)}
$$

Figure 4 shows the results obtained using several empirical and semi-empirical equations considering two-phase liquid-solid fluidized-bed expansion. Our own experimental data are also given.
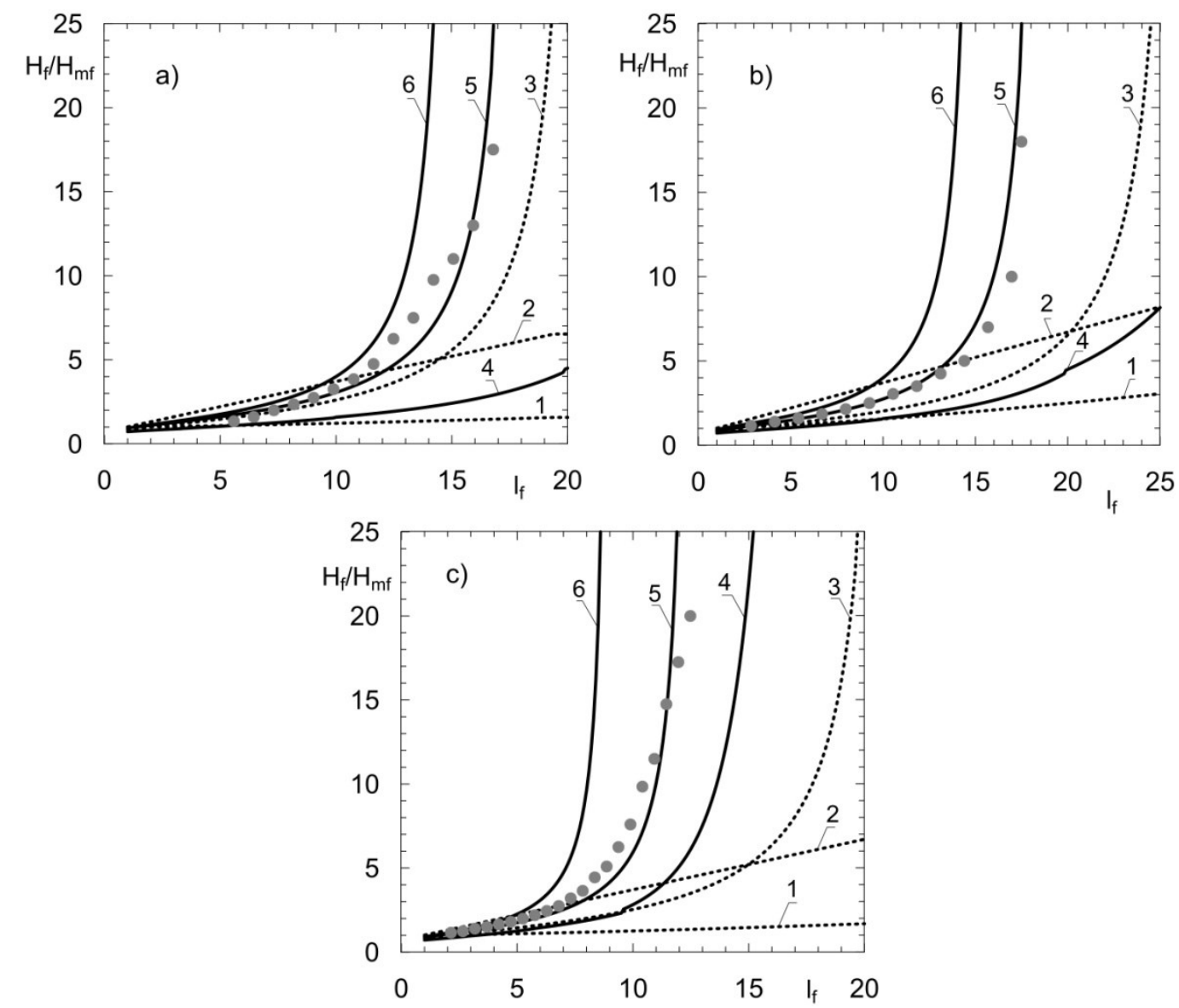

Fig. 4. Comparison of experimental and calculated bed expansion for two-phase liquid-solid fluidization (a- sand, $d_{s}=0.90 \mathrm{~mm}, \rho_{s}=2620 \mathrm{~kg} / \mathrm{m}^{3} ;$ b- coal, $d_{s}=0.90 \mathrm{~mm}, \rho_{s}=1412 \mathrm{~kg} / \mathrm{m}^{3} ; \mathrm{c}$ - brick,$d_{s}=0.90 \mathrm{~mm}$, $\left.\rho_{s}=2384 \mathrm{~kg} / \mathrm{m}^{3}\right) 1$ - Thripaty (2013); 2- Razumow (1975) $(a=0.715 ; b=0.30) ; 3$ - Kmieć (1980); 4- Leva (1959); 5- Dziubiński (2009); 6- Ergun (1952); • - experimental data

The graphs presented in Figs. $4 \mathrm{a}$ and $4 \mathrm{~b}$ indicate that for $l_{f}<10$ the experimental data $H_{f} / H_{m f}$ lie between the values calculated according to Razumow and Tripathy correlations. In Fig. $4 \mathrm{c}$ this condition is fulfilled for $l_{f}<5$. As the fluidization ratio increases, the calculated values of fluidized-bed expansion based on selected empirical correlations diverges more and more from the experimental data. The correlation proposed by Kmieć (1980) reflects the tendency of nonlinear rise in the fluidized-bed expansion along with an increasing fluidization ratio. However, this tendency is smaller than that obtained from the experimental data. None of the tested empirical relations predict exactly the increase in the fluidization-bed height for a high fluidization ratio, i.e. when superficial liquid velocity, $u_{0 c}$, approaches the terminal velocity of solid particles, $u_{t}$. On the other hand, all semi-empirical correlations reflect a nonlinear rise in the fluidized-bed height. The formula proposed by Dziubiński et al. (2009) seems to have the smallest errors for high values of $l_{f}$, therefore it was decided to use it in subsequent calculations. 
The results of calculations of hydrodynamic characteristics of the analyzed hybrid apparatus, according to the proposed model shown above, are presented in Fig. 5 and Fig. 6. These graphs were plotted for selected operating parameters, which are reported in Table 2.

Table 2. Values of parameters used for calculations of hydrodynamics characteristics

\begin{tabular}{|c|c|c|c|c|}
\hline Parameter & a) & b) & c) & Dimension \\
\hline$d_{1}$ & 0.2 & 0.2 & 0.2 & $\mathrm{~m}$ \\
\hline$d_{2}$ & 0.2 & 0.2 & 0.2 & $\mathrm{~m}$ \\
\hline$d_{3}$ & 0.25 & 0.23 & 0.25 & $\mathrm{~m}$ \\
\hline$H$ & 3 & 3 & 2 & $\mathrm{~m}$ \\
\hline$H_{r}$ & 1.5 & 1.5 & 1.5 & $\mathrm{~m}$ \\
\hline
\end{tabular}

For all analyzed cases values of the other parameters were the same, i.e.: $d_{s}=7 \cdot 10^{-4} \mathrm{~m} ; \rho_{s}=1800 \mathrm{~kg} \cdot \mathrm{m}^{-3}$; $H_{m f}=0.1 \mathrm{~m} ; \varepsilon_{m f}=0.5 ; \rho_{c}=1000 \mathrm{~kg} \cdot \mathrm{m}^{-3} ; \eta_{c}=0.001 \mathrm{~kg} \cdot \mathrm{m}^{-1} \cdot \mathrm{s}^{-1} ; \sigma_{c}=7.27 \cdot 10^{-2} \mathrm{~kg} \cdot \mathrm{s}^{-2}$.

Figure 5 shows the relationship between superficial liquid velocity in the zone number „1”, $u_{0 c 1}$, and superficial gas velocity $u_{0}$. The superficial velocity of liquid in this zone has two limits according to inequalities (1). These limits are marked in Fig. 5 by dotted lines. Figure 5a shows that for given dimensions of the bioreactor and according to Razumow's correlation (1975), it is possible to achieve two limit values $u_{0 \mathrm{~g}}$, for which $u_{0 c 1}=u_{m f}$ or $u_{0 c 1}=u_{t}$. When applying Dziubiński’s formula (2009), which is considered to be the most reliable, in the device of the same dimensions it is not possible to fulfill the equality $u_{0 c 1}=u_{t}$, because already at a lower superficial velocity, $u_{0 c 1}$, the other limiting value, corresponding to the bed height is satisfied (condition (2)).

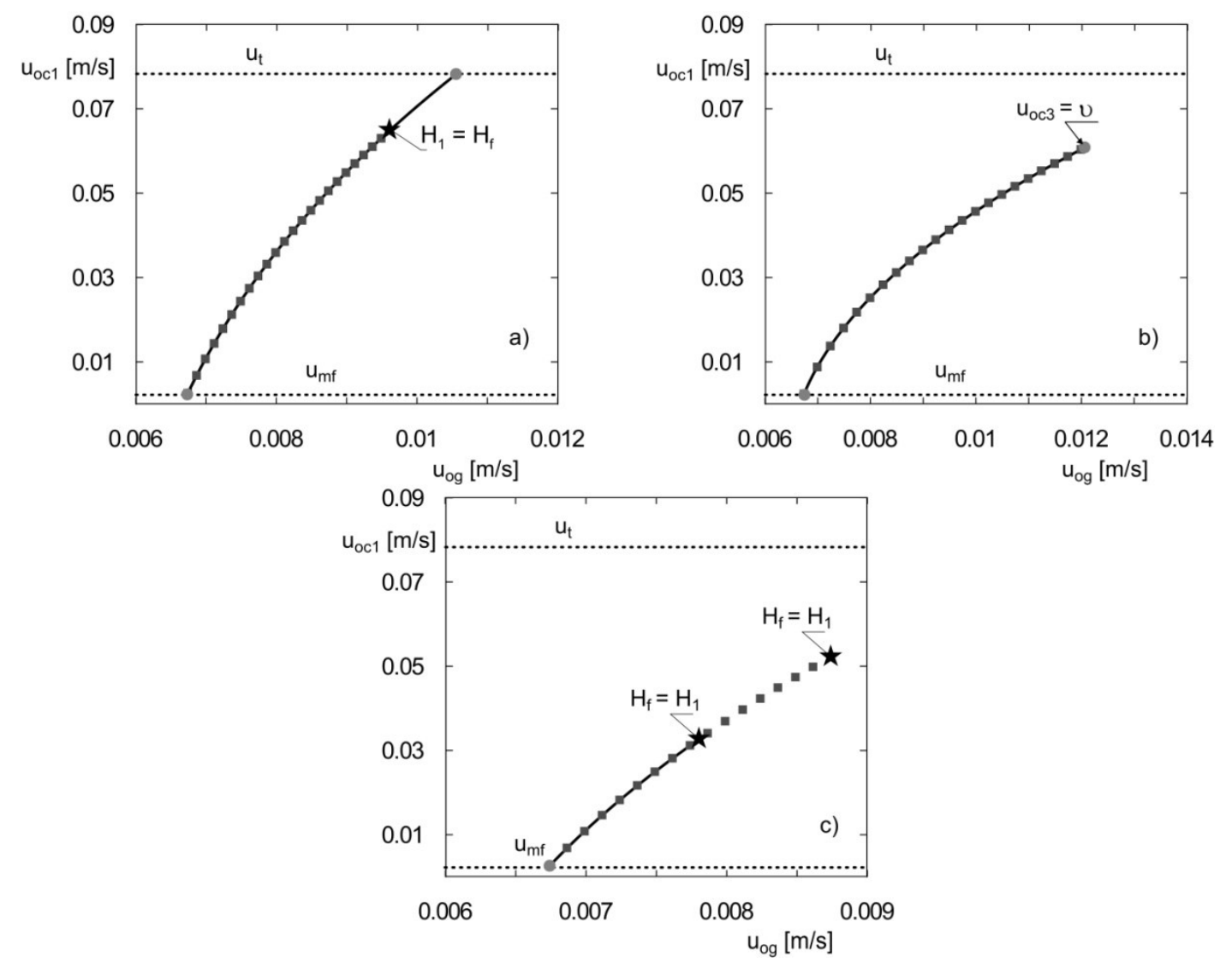

Fig. 5. Limit values of superficial gas velocity $u_{0 \mathrm{~g}}$ for various sizes of bioreactors

$$
\text { (- Razumow (1975); - Dziubiński (2009)) }
$$


If the column diameter, $d_{3}$, decreases, superficial liquid velocity in zone , $3 ", u_{03}$, can be higher than the slip velocity of gas bubbles. In this work it is assumed that there are no gas bubbles in zone „3”. Therefore, the condition $u_{0 c 3}=v$ determines the maximum value of superficial gas velocity, $u_{0 \mathrm{~g}}$. That limitation is shown in Fig. 5b. The application of Razumow`s (1975) and Dziubiński`s (2009) formulas gives similar results. Because of sufficient height of the device, despite bigger bed expansion calculated according to Dziubiński's formula (2009), the fluidized bed does not achieve the height of zone "1".

The graph presented in Fig. 5c corresponds to the situation when the upper limit value of superficial gas velocity, $u_{0 \mathrm{~g}}$, is determined by the height of zone number „1". An increase in superficial gas velocity, $u_{0 g}$, causes an increase in liquid circulation velocity in the device as well as expansion of the fluidized bed in zone "1". Thus for given dimensions of the reactor it is possible to determine the limit value of superficial gas velocity, for which $H_{f}=H_{1}$, where $H_{1}=H-H_{r}$. According to Dziubiński`s formula (2009) the limit bed height, $H_{f}=H_{1}$, was achieved for lower values of superficial liquid velocity, $u_{0 c 1}$, than those calculated from Razumow`s formula quoted before. The dynamic bed height calculated from Razumow's correlation is higher compared with experimental data for low fluidization ratios (Fig. 4). The analysis preformed here leads to the conclusion that the device works in the range of low values of fluidization ratio, $l_{f}$, for lower heights of zone " 1 ". Therefore, the application of Dziubiński"s formula (2009) is recommended because designing such devices seems to be much safer. This correlation was experimentally verified in a wide range of fluidization ratios by the authors.

Maximum and minimum superficial gas velocities, $u_{0 g}$, which ensure effective utilization of the fluidized-bed part of the apparatus, depend on physical properties of liquid, gas and solids. In Fig. 6 the mentioned limit values of superficial gas velocity, as two functions of Archimedes number for a device with given dimensions i.e. $H, H_{r}$ and $d_{3}$ are shown. The upper curve corresponds to the values of $u_{0 g}$, for which the superficial liquid velocity, $u_{0 c 1}$, in the fluidization zone is equal to the terminal velocity of solids, i.e. $u_{0 c 1}=u_{t}$. The lower curve corresponds to the values of $u_{0 g}$, for which $u_{0 c 1}=u_{m f}$. The limit curves presented in Fig. 6 may be considered as a basis for designing such hybrid devices. The lower limit of superficial velocity is appropriate for an apparatus with any height of fluidization zone, $H_{1}$. On the other hand, the upper limit should be taken into consideration for a high enough fluidization zone which satisfies condition (2). For this reason it may be used for evaluation of the maximum height of this zone while designing such devices.

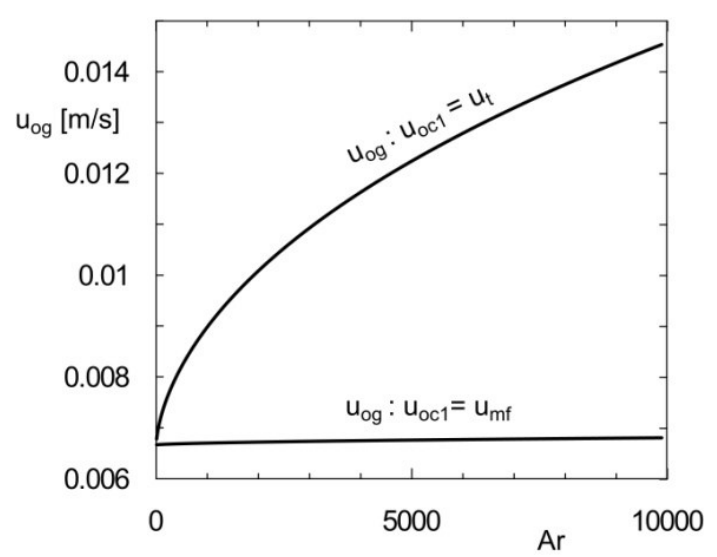

Fig. 6. Lower and upper limits of superficial gas velocity versus Archimedes number $\left(H=3 \mathrm{~m}, H_{r}=1.5 \mathrm{~m}, d_{1}=0.2 \mathrm{~m}, d_{2}=0.2 \mathrm{~m}, d_{3}=0.25 \mathrm{~m}\right)$

\section{CONCLUSIONS}

Hydrodynamic characteristics of a hybrid apparatus consisting of an air-lift and two-phase fluidizedbed parts is presented. The application of this type of hybrid devices working as bioreactors brings 
about higher biomass concentration and eliminates main problems arising from the use of three-phase fluidization. However, splitting the apparatus into two parts makes it possible to work only within a specific range of operating and geometrical parameters. It is caused by hydrodynamic limitations tied up with proper work of such devices.

For the purpose of simulating and designing hybrid fluidized-bed bioreactors it is necessary to create simple and sufficiently accurate equations for momentum and mass balances of flowing media. This paper presents equations that allow to assess the operating parameters influencing hydrodynamics of such devices. The presented hydrodynamic model facilitates the proper selection of basic reactor geometry, i.e. diameters and heights of particular zones. When the analysis of a reactor existing in a real installation is performed, we can determine the ranges of operating parameters, wherein the apparatus may work correctly, i.e. as a hybrid device.

In this work, selected and representative correlations for calculation of fluidized-bed expansion are compared. Our work contains only a few correlations arisen in 1952- 2013. Correct determination of the dynamic height of fluidized bed is an essential element during the process design, numerical simulations, and technological practice. Incorrect matching of the fluidized bed height makes it impossible for the apparatus to work. If the height of this zone is insufficient, fine-grained solid will accumulate under the upper net. This will result in the presence of a fixed bed instead of a fluidized bed. On the other hand, an excessive increase of the height of this zone brings about the increase of pressure drop tied up with medium flow and it could make liquid circulation impossible.

Hydrodynamic analysis of hybrid fluidized-bed bioreactors is important because of the possibility of utilizing these reactors in many aerobic bioprocesses. The appropriate selection of hydrodynamic and geometrical parameters is the basis for modeling and designing microbiological processes in such bioreactors.

\section{SYMBOLS}

$\begin{array}{ll}A r & \text { Archimedes number } \\ A & \text { surface area, } \mathrm{m}^{2} \\ \left(C_{x}\right)_{u} & \text { drag coefficient } \\ d & \text { diameter, } \mathrm{m} \\ F_{V}^{g}, F_{V}^{c} & \text { volumetric flow rate of gas and liquid, respectively, } \mathrm{m}^{3} / \mathrm{s} \\ g & \text { gravity acceleration, } \mathrm{m} / \mathrm{s}^{2} \\ H & \text { total height of the bioreactor, } \mathrm{m} \\ H_{f} & \text { dynamic height of the fluidized bed, } \mathrm{m} \\ H_{r} & \text { height of the riser, } \mathrm{m} \\ l_{f} & \text { fluidization ratio, } l_{f}=u_{0 c} / u_{m f} \\ l_{f m i n}, l_{f t} & \text { fluidization ratio corresponding } u_{m f} \text { and } u_{t}, \text { respectively } \\ n & \text { index parameter }, n=f(\text { Re } s) \\ \Delta p & \text { pressure drop, Pa } \\ S & \text { cross sectional area, } \mathrm{m}^{2} \\ u_{c}, u_{g} & \text { velocity of liquid and gas, } \mathrm{m} / \mathrm{s} \\ u_{m f} & \text { minimum fluidization velocity, } \mathrm{m} / \mathrm{s} \\ u_{t} & \text { terminal velocity of solid particles, } \mathrm{m} / \mathrm{s} \\ V & \text { volume, } \mathrm{m}^{3} \\ & \end{array}$




$\begin{array}{ll}\eta & \text { viscosity, } \mathrm{kg} /(\mathrm{m} \cdot \mathrm{s}) \\ \lambda & \text { friction factor } \\ \rho & \text { density, } \mathrm{kg} / \mathrm{m}^{3} \\ \sigma & \text { surface tension, } \mathrm{kg} / \mathrm{s}^{2} \\ \nu & \text { slip velocity of gas bubbles, } \mathrm{m} / \mathrm{s} \\ \Phi & \text { shape coefficient }\end{array}$

\section{Subscripts}

1, 2, 3 refers to a particular zone (see Fig.2b)

0 values referring to the cross sectional area of a particular zone

$b \quad$ surroundings of the lower edge of the riser

$c \quad$ liquid

$f \quad$ refers to fluidized bed

$k$ column

$m f \quad$ refers to minimum fluidization conditions

$r \quad$ riser

$s \quad$ solid

$t \quad$ refers to terminal velocity of solid particles

\section{REFERENCES}

Dunn I.J., Tanaka H., Uzman S., Denac M., 1983. Biofilm fluidized-bed reactors and their application to waste water nitrification. Ann. NY Acad. Sci., 413, 168-183. DOI: 10.1111/j.1749-6632.1983.tb47887.x.

Dunn I.J., Heinzle E., Ingham J., Prenosil J.E., 2003. Biological reaction engineering. Dynamic modelling fundamentals with simulation examples. Wiley-VCH Verlag, Weinhem.

Di Felice R., 1995. Hydrodynamics of liquid fluidisation. Chem. Eng. Sci., 50, 1213-1245. DOI: 10.1016/00092509(95)98838-6.

Dziubiński M., Prywer J., 2009. Two-phase fluid mechanics (Mechanika płynów dwufazowych). WNT, Warszawa, Poland.

Dziubiński M., Sowiński J., 1999. The specific interfacial area in an air-lift column (in Polish). Chem. Proc. Eng., 20(3), 409-421.

Dziubiński M., Sowiński J., 2002. Liquid circulation velocity in pilot-plant scale air-lift columns (in Polish). Chem. Proc. Eng., 23, 141-150.

Ergun S., 1952. Fluid flow through packed columns. Chem. Eng. Prog., 48, 89-94.

Garnier A., Chavarie C., Andre G., Klvana D., 1990. The inverse fluidization airlift bioreactor, Part I: hydrodynamic studies. Chem. Eng. Comm., 98 , 31-45. DOI: 10.1080/00986449008911559.

Grzywacz R., 2013. Airlift Bioreactor. Wydawnictwo Politechniki Krakowskiej, Kraków, Poland.

Guo Y.X., Rathor M.N., Ti H.C., 1997. Hydrodynamic and mass transfer studies in a novel external-loop airlift reactor. Chem. Eng. J., 67, 205-214. DOI: 10.1016/S1385-8947(97)00043-0.

Heijnen J.J., Hols J., Van der Lans R.G.J.M., Van Leeuwen H.L.J.M., Mulder A., Welte-vrede R., 1997. A simple hydrodynamic model for the liquid circulation velocity in a full scale two- and three-phase internal airlift reactor operating in the gas recirculation regime. Chem. Eng. Sci., 52, 2527-2540. DOI: 10.1016/S0009-2509(97)000705 .

Huang Y.L., Shu C.H., Yang S.T., 1997. Kinetics and modeling of GM-CSF production by recombinant yeast in 3-phase fluidized-bed bioreactor. Biotechnol. Bioeng., 53, 470-477. DOI: 10.1002/(SICI)10970290(19970305)53:5<470:AID-BIT4>3.0.CO;2-E.

Kawalec- Pietrenko B., 2000. Liquid circulation velocity in the inverse fluidized bed airlift reactor. Bioproc. Eng., 23, 397-402. DOI: 10.1007/s004499900182.

Kawalec- Pietrenko B., 2004. Three-phase airlift reactors (in Polish). Chem. Proc. Eng., 25, 1925-1935. 
Kawalec- Pietrenko B., Holowacz I., 1998. Region-dependent oxygen transfer rate in the rectangular airlift reactor. Bioproc. Eng., 18, 163-170. DOI: 10.1007/s004490050426.

Kawalec- Pietrenko B., Łazarczyk M., 2004. Application of an air-lift reactor with the inverse fluidized-bed in biodegradation of dimethyl ketone (in Polish). Inż. Ap. Chem., 2, 3-5.

Kawalec- Pietrenko B., Matczak B., 2006. Comparison of the oxygen transfer rate in the inverse fluidized-bed airlift reactor and related bubble column reactors (in Polish). Chem. Proc. Eng., 27, 125-139.

Kmieć A., 1980. Bed expansion and heat and mass transfer in fluidized beds (in Polish). Scientific papers of the Institute of Chemical Engineering and Heating Equipment, Wrocław, Poland.

Leva M., 1959. Fluidization, Mc Graw-Hill, New York.

Livingston A.G., 1991. Biodegradation of 3,4-dichloroaniline in a fluidized bed reactor and a steady state biofilm kinetic model. Biotechnol. Bioeng., 38, 260-272. DOI: 10.1002/bit.260380308.

Lu W.J., Hwang S.J., Chang C.M., 1995. Liquid velocity and gas holdup in three-phase internal loop airlift reactors with low-density particles. Chem. Eng. Sci., 50, 1301-1310. DOI: 10.1016/0009-2509(95)98842-3.

Merchuk J.C., 2003. Airlift bioreactors: review of recent advances. Can. J. Chem. Eng., 81, 324-337. DOI: 10.1002/cjce.5450810301.

Merchuk J.C., Siegel M.H., 1988. Airlift reactors in chemical and biological technology. J. Chem. Tech. Biotechnol., 41, 105-120. DOI: 10.1002/jctb.280410204.

Miyahara T., Kawate O., 1993. Hydrodynamics of a solid-suspensed bubble column with a draught tube containing low density particles. Chem. Eng. Sci., 48, 127-133. DOI: 10.1016/0009-2509(93)80289-3.

Mowla D., Ahmadi M., 2007. Theoretical and experimental investigation of biodegradation of hydrocarbon polluted water in a three phase fluidized-bed bioreactor with PVC biofilm support. Biochem. Eng. J. 36, 147156. DOI: $10.1016 /$ j.bej.2007.02.031.

Nore O., Briens C., Margaritis A., Wild G., 1992. Hydrodynamics, gas-liquid mass transfer and particles-liquid heat and mass transfer in a three-phase fluidized bed for biochemical process applications. Chem. Eng. Sci., 47, 3573-3580. DOI: 10.1016/0009-2509(92)85072-J.

Olivieri G., Marzocchella A., Salatino P., 2010. A novel three-phase airlift reactor without circulation of solids. Can. J. Chem. Eng., 88, 574-578. DOI: 10.1002/cjce.20314.

Onysko K.A., Robinson C.W., Budman H.M., 2002. Improved modelling of the unsteady-state behavior of an immobilized-cell, fluidized-bed bioreactor for phenol biodegradation. Can. J. Chem. Eng., 80, 239-252. DOI: 10.1002/cjce.5450800209.

Razumow I.M., 1975. Fluidization and pneumatic transport of fine materials (in Polish). WNT, Warszawa.

Sarra M., Casas C., Godia F., 1997. Continuous production of a hybrid antibiotic by Streptomyces-lividans Tk21 pellets in a 3-phase fluidized-bed bioreactor. Biotechnol. Bioeng., 53, 601-610. DOI: 10.1002/(SICI)1097-0290.

Tabiś B., Georgiou A., 2003. Method for the determination of the steady states of a three-phase fluidized-bed bioreactor. Chem. Proc. Eng., 24, 551-566.

Tabiś B., Kupiec K., 2003. Hydrodynamics of a three-phase airlift bioreactor containing low-density particles (in Polish). Chem. Proc. Eng., 24, 217-233.

Tabiś B., Stryjewski W., 2013. Conditions for application of fluidized-bed bioreactors in aerobic processes (in Polish). Inż. Ap. Chem. 52, 487-489.

Tang W.-T., Fan L.-S., 1987. Steady state phenol degradation in a draft-tube, gas-liquid-solid fluidized-bed bioreactor. AICHE J. 33, 239-249. DOI: 10.1002/aic.690330210.

Tang W.T., Wisecarver K., Fan L.S., 1987. Dynamics of a draft tube gas-liquid-solid fluidized bed bioreactor for phenol degradation. Chem. Eng. Sci., 42, 2123-2134. DOI: 10.1016/0009-2509(87)85033-9.

Tripathy A., Sahu A.K., Biswal S.K., Mishra B.K., 2013. A model for expansion ratio in liquid-solid fluidized beds. Particuology. 11, 789-792. DOI: 10.1016/j.partic.2012.11.006.

Vunjak-Novakovic G., Jovanovic G., Kundakovic L., Obradovic B., 1992. Flow regimes and liquid mixing in a draft tube gas-liquid-solid fluidized bed. Chem. Eng. Sci., 47, 3451-3458. DOI: 10.1016/0009-2509(92)85057-I.

Wisecarver K.D., Fan L.S., 1989. Biological phenol degradation in a gas-liquid-solid fluidized bed reactor. Biotechnol. Bioeng., 33, 1028-1038. DOI: 10.1002/bit.260330812. 